\title{
ULF impulsive magnetic response at mid-latitudes to lightning activity
}

\author{
A. Schekotov ${ }^{1}$, V. Pilipenko ${ }^{1}$, K. Shiokawa ${ }^{2}$, and E. Fedorov ${ }^{1}$ \\ ${ }^{1}$ Institute of the Physics of the Earth, Moscow \\ ${ }^{2}$ STEL, Nagoya University, Japan
}

(Received August 31, 2010; Revised December 1, 2010; Accepted December 14, 2010; Online published February 28, 2011)

\begin{abstract}
Induction magnetometer data from the mid-latitude station Moshiri (geomagnetic latitude $35.6^{\circ}$ ) has been examined in search of a transient ULF response to the regional lightning activity. For many events, besides the main impulse produced by the lightning discharge, a secondary impulse delayed about $1 \mathrm{sec}$ was observed. These secondary echo-impulses are probably caused by the partial re ection of wave energy of the initial lightning pulse from the upper boundary of the ionospheric Alfven resonator in the topside ionosphere. The modeling with arti cial signals has shown that a multi-band spectral resonant structure (SRS) can be formed owing to the occurrence of paired pulses in analyzed time series. The statistical superposed epoch method indeed has revealed a dominance of two-pulse structure in the magnetic eld background during the periods of the SRS occurrence.

Key words: Lightning, Pc1 waves, ionosphere, Alfven resonator, spectral resonant structure.
\end{abstract}

\section{Introduction}

Electrical storms are known to be one of the natural ULF sources in the Earth's atmosphere. Besides the well-known global excitation of the Earth-ionosphere cavity Schumann resonances (SR) in the ELF frequency band of several tens $\mathrm{Hz}$ (Nickolaenko and Hayakawa, 2002), local lightning transients and large ULF noise enhancement in the frequency range of several $\mathrm{Hz}$ in the thunderstorm proximity were observed (Fraser-Smith, 1993). A peculiar feature of the geomagnetic eld variations in the ULF band just below the fundamental tone of SR is the occurrence of Spectral Resonant Structure (SRS), observed at low (Bösinger et al., 2002), middle (Belyaev et al., 1987, 1990) and high (Belyaev et al., 1999; Yahnin et al., 2003) latitudes. The lowest frequency of the SRS was about fraction of $\mathrm{Hz}$, and the difference between the equidistant spectral harmonics was about $0.3-0.5 \mathrm{~Hz}$. Analysis of the SRS at mid-latitude demonstrated evident seasonal variation in the occurrence rate with the maximum at the September-January period and almost complete absence of SRS at the spring-early summer time (Molchanov et al., 2004). The SRS is the nighttime phenomenon, almost all the SRS were observed between 19 and 05 LT.

The occurrence of SRS was commonly attributed to the Ionospheric Alfven Resonator (IAR) in the upper ionosphere (Polyakov and Rapoport, 1981; Demekhov et al., 2000). The IAR lower boundary coincides with the ionospheric bottomside boundary, whereas the upper boundary is located at altitude of few thousands $\mathrm{km}$ due to the partial re ection of the Alfven waves from a steep gradient of the Alfven velocity vertical pro le $V_{\mathrm{A}}(z)$ above the maximum

Copyright (C) The Society of Geomagnetism and Earth, Planetary and Space Sciences (SGEPSS); The Seismological Society of Japan; The Volcanological Society of Japan; The Geodetic Society of Japan; The Japanese Society for Planetary Sciences; TERRAPUB.

doi:10.5047/eps.2010.12.009 of the $F$-layer. The damping rates and $Q$-factors of the IAR modes strongly depend on the Alfven velocity differential in the upper ionosphere and on the lower ionosphere conductivity.

The ionospheric cavity with minimum of $V_{\mathrm{A}}(z)$ works not only as a resonator for Alfven waves, but as a waveguide for the magnetosonic mode also. The waveguide magnetosonic modes excited by the magnetospheric (Fujita and Tamao, 1988) or atmospheric (Pilipenko et al., 2005) sources can propagate to large distances (up to few thousand $\mathrm{km}$ ) along the ionosphere. In the frequency domain the ionospheric wave guidance manifests itself as an occurrence of low-frequency cut-off corresponding to the waveguide critical frequency (lower than the IAR fundamental frequency).

While the spectral properties of the IAR are well reproduced by numerous analytical and numerical models (e.g., Prikner et al., 2000), a physical mechanism of the IAR excitation has not been found yet. At high latitudes the mechanism of resonant energy transmission from the magnetospheric convective ows to the IAR normal modes may be operative (Trakhtengertz and Feldstein, 1991). The "feedback instability" due to the ionospheric modi cation by the energetic electron precipitation can also stimulate the IAR (Lysak, 1991; Pokhotelov et al., 2001). Evidently, these mechanisms can operate at auroral latitudes only. Moreover, the observed anti-correlation between the SRS occurrence rate and $K_{\mathrm{p}}$ index at mid-latitudes indicates a nonmagnetospheric origin of the SRS (Molchanov et al., 2004).

As a possible source of the IAR excitation, the generation of the ionospheric turbulence and currents by the neutral wind in the lower ionosphere has been suggested (Surkov et al., 2004). These authors showed theoretically that a part of the neutral gas kinetic energy can be transferred into the energy of the electric current in the conductive ionosphere, which is then converted into the energy of Alfven and fast 


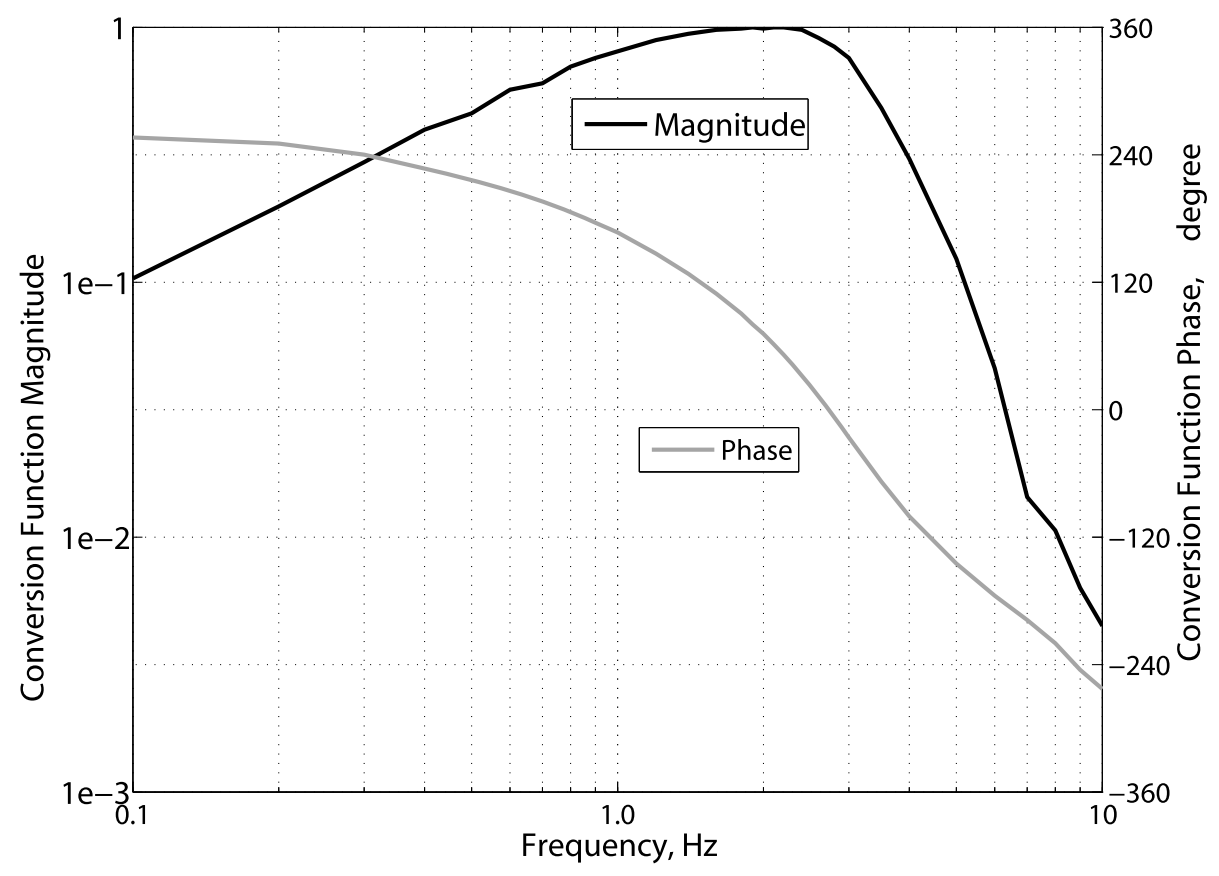

Fig. 1. The conversion function (magnitude and phase) of the induction magnetometer and data acquisition system.

compressional MHD modes. However, this hypothesis has not been experimentally validated yet.

The other possible energy source for the IAR excitation is related to lightning discharges. The world thunderstorm centers were suggested to be the primary source of the IAR excitation (Polyakov and Rapoport, 1981; Belyaev et al., 1987; Bösinger et al., 2002). However, theoretical estimates have shown that the contribution of the distant world thunderstorm centers with typical charge moments of negative CG- flashes to the mid-latitude electromagnetic field in the IAR band is about two orders of magnitude less than the observed intensity (Fedorov et al., 2006). However, Shalimov and Bösinger (2008) proposed that relatively infrequent $(\sim 10 \%)$, but more intense (about an order of magnitude) positive cloud-to-ground flashes $\mathrm{CG}+$ can provide a necessary ULF background at large distances. Thus, a possible role of distant thunderstorms in the IAR stimulation is still disputable.

As an alternative mechanism, it was suggested that nearby thunderstorms are able to generate the signals in the IAR range with sufficient intensities (Surkov et al., 2006). In this paper we examine the latter hypothesis and search for a ULF magnetic field response to the regional lightning activity, using data from the high-sensitive induction magnetometer at the station Moshiri. Our analysis has shown that the mechanism of the SRS formation is not related to the oscillatory response of the IAR, as commonly assumed, but is caused by the specific multi-pulse structure of geomagnetic disturbances during the lightning activity.

\section{Data Description}

For our study we used the ULF magnetic field recordings from Moshiri observatory (MOS, geographical coordinates $44.37^{\circ} \mathrm{N}, 142.27^{\circ} \mathrm{E}$, geomagnetic coordinates $\Phi=35.6^{\circ}$, $\Lambda=209.5^{\circ} ; L=1.6$, JST $=$ UT +9 ) in Japan, de- ployed by Solar-Terrestrial Environment Laboratory of the Nagoya University (Shiokawa et al., 2010). Three-axial induction magnetometer ( $H$-component is oriented in the N-S magnetic direction, southward positive; $D$ is oriented towards the west direction, westward positive; and vertical $Z$ component is positive downward) measures magnetic field variations with the sampling frequency $64 \mathrm{~Hz}$ periodically synchronized by GPS clock signal. All component sensors have basically the same specifications: turnover frequency $2.3-2.9 \mathrm{~Hz}$, conversion factor $13.2 \mathrm{~V} / \mathrm{nT}$ at $2.3-2.9 \mathrm{~Hz}$, and $1.27 \mathrm{~V} / \mathrm{nT}$ at $0.1 \mathrm{~Hz}$. The amplitude-phase frequency response of the data acquisition system (Fig. 1) has a very steep decline at higher frequencies, above $\sim 3 \mathrm{~Hz}: 2.5$ orders of magnitude in the range from $3 \mathrm{~Hz}$ to $10 \mathrm{~Hz}$ to suppress the influence of commercial power line noise at $60 \mathrm{~Hz}$.

We have analyzed the time intervals with evident SRS in the sonograms from MOS station during October 2007. For three 1-hour time intervals we have the information on regional lighting strokes from the lightning detection system (timing, location, intensity and type of a stroke) purchased from the Japan Lightning Detection Network operated by Franklin Japan Co., Ltd. The available list of lightning events comprises strokes within the $100 \mathrm{~km}$ circle around a point located $300 \mathrm{~km}$ southward of MOS. The near-by lightning (about $100 \mathrm{~km}$ and less from the observation site) produce too strong interference on magnetic records, and the possible SRS features cannot be revealed.

\section{Lightning-related Impulses: Case Events}

For many events an impulse produced by a lightning discharge can be seen in the magnetometer data. These impulses are more evident in the raw data from the induction sensors, without correction of the frequency transfer characteristics. As a typical example, the response to the stroke on Oct. 29 is shown in Fig. 2. The negative stroke with 
Lightning time: $22 \mathrm{~h} 53^{\prime} 42^{\prime \prime} 29 / 10 / 2007$
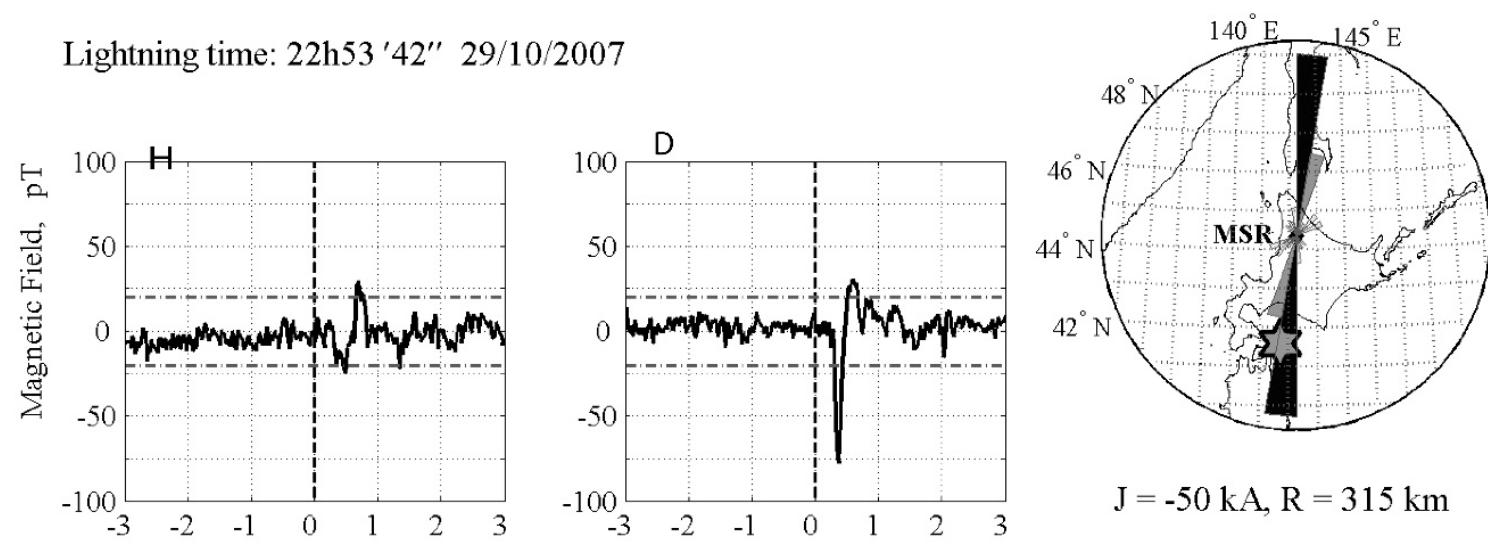

Fig. 2. The example of the ULF impulse for the stroke on $10 / 29,22: 53: 42(J=-50 \mathrm{kA}, R=315 \mathrm{~km})$ detected by the induction magnetometer at MOS: wave forms of $H$ and $D$ components. Right-hand insert shows the map with location of lightning, marked by star. The length and darkness of a lobe on the circular diagram corresponds to the intensity of the pulse energy ow.

the effective intensity $J=-50 \mathrm{kA}$, identi ed by the lightning monitoring system at 22:53'42" LT, took place at distance $R=315 \mathrm{~km}$ (marked by the dashed vertical line). At this moment a sharp impulse can be seen, especially in $D$-component. Its amplitude, $\sim 70 \mathrm{pT}$, has been roughly estimated as the sensor output multiplied by the ampli cation coef cient at frequency $2 \mathrm{~Hz}$. A weak oscillatory response at $f \sim 2-3 \mathrm{~Hz}$ just after the main impulse is due to the peculiarities of the frequency response function.

A source location has been determined with the use of polarization analysis, assuming that the main axis of the polarization ellipse is to be perpendicular to the direction towards a source (the goniometer method). The orientation of polarization ellipse, that is the angle $\theta$ that the principle axis of polarization ellipse makes with W-E direction, is estimated by the formula (Fowler et al., 1967):

$$
\tan 2 \theta=\frac{2 \operatorname{Re} P_{\mathrm{hd}}}{P_{\mathrm{hh}}-P_{\mathrm{dd}}}
$$

where $P_{\mathrm{hh}}, P_{\mathrm{dd}}$, and $P_{\mathrm{hd}}$ are the spectral densities and crossspectral density calculated by the wavelet transform (3-d order complex Gauss mother wavelet). Finally, the signal source azimuth angle $\alpha$ is determined as $\alpha=180-\theta$.

The range of possible directions to the source, identi ed with the polarization technique, is shown in Fig. 2 (righthand panel). The length and color of a lobe on the circular pie diagram corresponds to the squared intensity of the impulse energy ow in a given angular sector. The identied direction corresponds well to the actual location of the stroke (marked by star). The threshold level for the signal detection was chosen to be 10 times of root mean square of background uctuations (shown by horizontal dashed lines). For such signals with the signal/noise ratio above 10 the error of the source azimuth determination is better than $6^{\circ}$.

\section{Impulsive Magnetic Background}

For many events a more complicated pattern is observed (Fig. 3). Although for most of them we do not have information from the lightning detection system, the morphological features of these pulses evidence that they have been produced by lightning strokes. For a typical event on Oct. 4 at 22:07:26.4 LT the main pulse is followed by the secondary impulse in $D$-component. The delay between the main and secondary impulses is $\Delta t \sim 1 \mathrm{~s}$. This series of two-impulses results in the multi-band structure in the corresponding spectra (Fig. 3, bottom panels).

Besides the standard dynamic and static Fourier spectra, we have employed the wavelet spectral estimates. Most of signals in the considered frequency band are transient or short-lived, and for such signals the wavelet transform is superior to the Fourier transform. As a mother wavelet the complex Gaussian or complex Morlet wavelet has been used. The choice of wavelet and its order has been made as a compromise between the frequency and time resolution. Both wavelet and time-integrated wavelet spectra (Fig. 3, right-hand panels) show the occurrence of spectral peaks at $f \sim 1.5 \mathrm{~Hz}$, and $2.5 \mathrm{~Hz}$. The frequency shift between the spectral lines, $\Delta f \sim 1 \mathrm{~Hz}$, corresponds to the inverse time delay between impulses, $\Delta f \sim 1 / \Delta t$.

Multi-harmonic SRS of ULF pulsations in the frequency range $0.5-5 \mathrm{~Hz}$ during nighttime are commonly associated with the periodic response of the IAR to an external driving. However, a thorough examination of magnetometer records during the SRS occurrence shows the absence of the narrow-band oscillations, similar to SR. The further analysis will show that in fact the multi-harmonic SRS is produced not by quasi-periodic oscillations, but by a regular sequence of 2, sometimes even 3, impulses.

For the scrutiny analysis we have selected the day Oct. 4 with the SRS occurrence (Fig. 4). Below the SR emission at $\sim 8 \mathrm{~Hz}$, one can see the SRS, especially evident in $D$ component, from $\sim 19$ LT to $\sim 06$ LT.

To visualize the average waveform of the ULF disturbance, the program developed selects in a speci ed interval all impulses above a certain threshold separately for $H$ and $D$ components in a running time window. Then, all the waveforms are averaged over the time interval $\pm 3 \mathrm{~s}$, using the superposed epoch method, choosing as a reference time the moment of a pulse peak value. To examine the impulsive structure of the ULF activity this time window has been chosen to be larger than the expected time delay between 

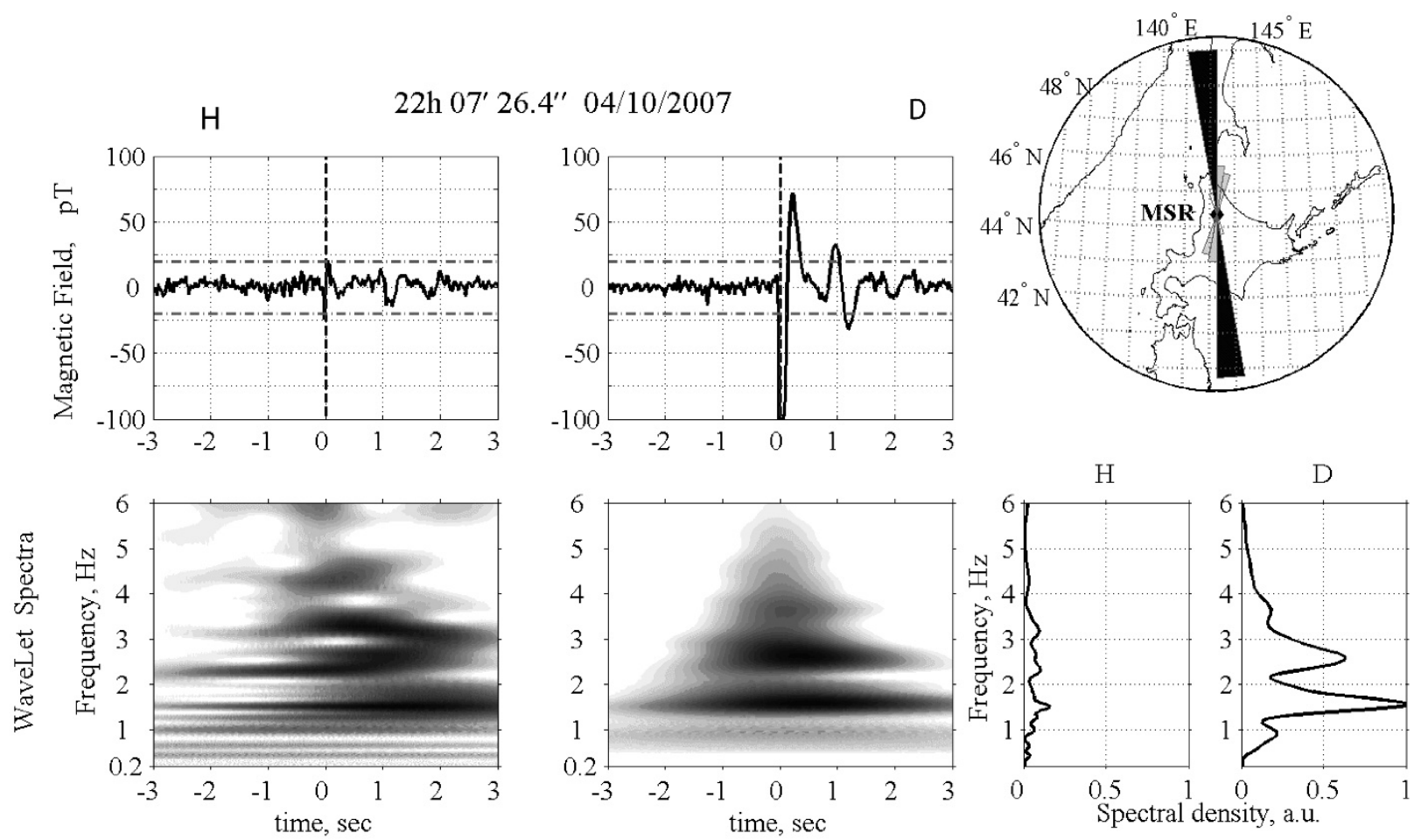

Fig. 3. The example of two-pulses pattern observed on 10/04 22:07:26.4 LT (stroke moment is marked by a vertical dashed line) at MOS. The upper panels show waveforms of $H$ (left-hand) and $D$ (right-hand) components, and bottom panels show the relevant wavelet spectra. Time-averaged wavelet spectra are shown in the right-hand bottom panel. Right-hand upper insert shows the map with direction towards a source.
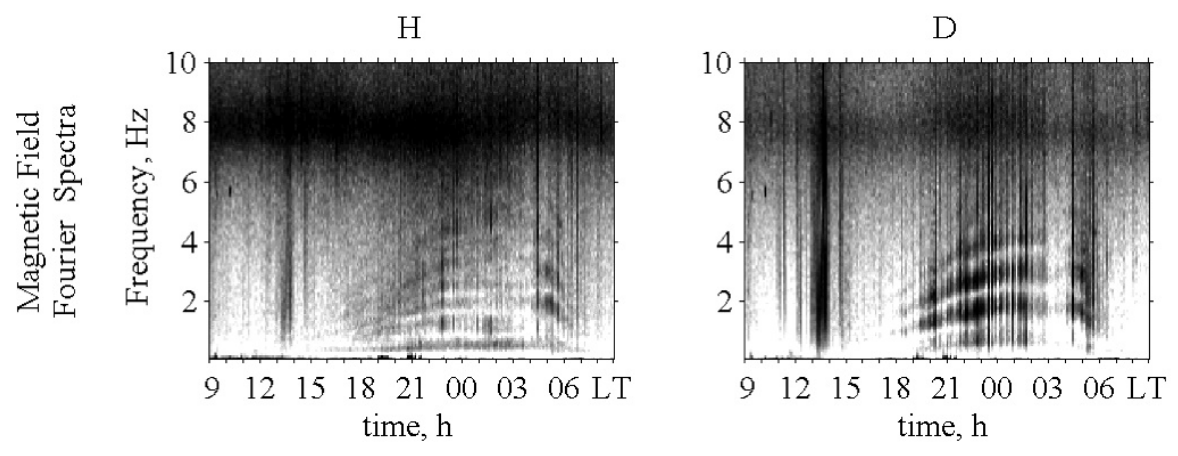

Fig. 4. The dynamic spectra for the period with the SRS occurrence at MOS on 10/04 for both horizontal magnetic components.

the main and subsequent pulses $(\sim 1 \mathrm{~s})$ and short enough to avoid the contamination by pulses from different lightning strokes.

The result of the superposed epoch analysis of the impulsive background during the hourly interval on Oct. 4, 22-23 LT is shown in Fig. 5. The upper panel shows the averaged impulse waveform for this time interval. Although the number of impulses is very large, $N_{\mathrm{av}}=270$, the timeaveraged impulsive background demonstrate a regular pattern: the main bipolar impulse is followed by the secondary bipolar impulse. The energy (squared amplitude on bottom panel) of the secondary pulse is about 25 smaller than that of main pulse. The third echo-pulse cannot be reliably revealed for this event.

To examine the time evolution of the impulsive background and SRS, Fig. 6 shows for six consecutive hourly intervals the quasi-3D dynamic Fourier spectra (upper pan- els), averaged Fourier spectra (mid-panels), and average waveforms of superposed impulses (bottom panels) for Oct. 4 from 18 LT to 24 LT. This plot demonstrates a close correspondence between the temporal evolution of the impulsive background in $D$-component and SRS. During the time interval 18-19 LT there are no SRS and no noticeable impulses. On 19-20 LT a weak indication on SRS occurrence has emerged, and, simultaneously, a 2-impulse structure has started to be formed. On 20-24 LT the SRS evolves and intensi es, whereas multi-impulse structure has become evident. The average time delay and the spectral gap between harmonics also vary in a consistent way: on 19-20 LT $\Delta t \sim 1.2 \mathrm{~s}$ and $\Delta f \sim 0.8 \mathrm{~Hz}$, whereas on 22$23 \mathrm{LT} \Delta t \sim 0.9 \mathrm{~s}$ and $\Delta f \sim 1.1 \mathrm{~Hz}$. The spectra minima are multiples of a fundamental frequency, on 19-20 LT $f \sim 0.8,1.6, \ldots \mathrm{Hz}$, whereas on 22-23 LT $f \sim 1.1,2.2$, $3.3, \ldots \mathrm{Hz}$. The averaged waveforms show a dominance of 

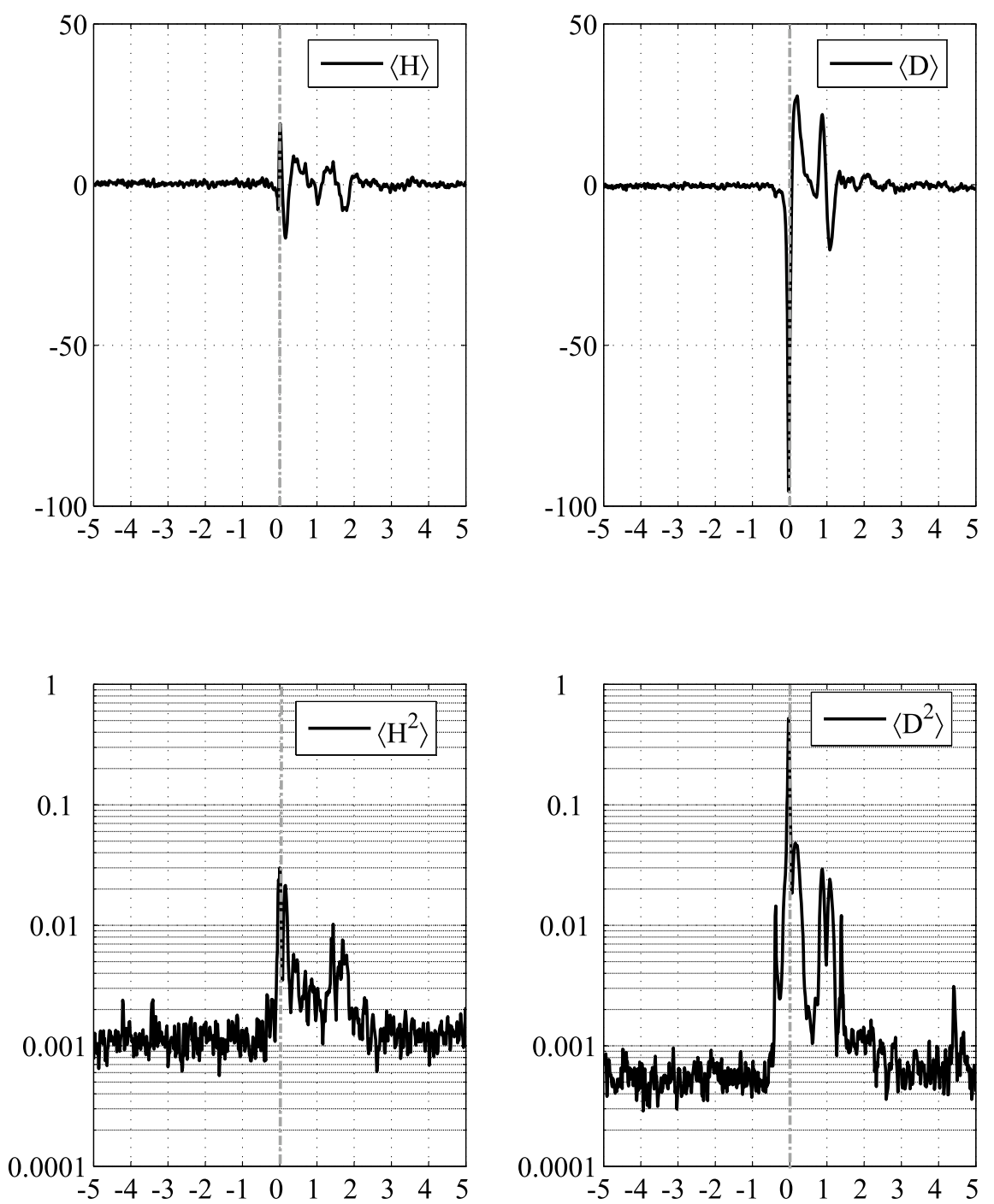

Fig. 5. Hourly superposed waveforms in the 10-sec vicinity (top panels) and superposed squared waveforms (bottom panels) of detected impulses on Oct. 4, 21-22 LT for $H$ (left-hand panels) and $D$ (right-hand panels) components. $N_{\mathrm{AV}}$ is the number of detected pulses during analyzed interval.

negative $D$-component pulses, which indicate the location of lightning activity southward from the station.

A similar characteristic multi-pulse background structure has been observed during all other intervals with evident SRS on October, 2007. This feature has been verified with the statistical superposed epoch method.

\section{Comparison of Multi-pulse Structure in the IAR and SR Bands}

The occurrence of subsequent magnetic pulses after a main pulse may be related, in principle, by multiple strokes during an atmospheric electric discharge. In order to examine this possibility, we have compared the time structure of the magnetic background in the SRS band $(0.3-3 \mathrm{~Hz})$ and SR band $(6-15 \mathrm{~Hz})$. For the statistical analysis we have applied the same superposed epoch technique as before. Figure 7 shows the statistically averaged magnetic response in the SR (upper panel) and SRS (lower panel) bands for both components on Oct. 04, 21-22 LT. The waveforms of pulses differ from those in previous figures, because here we have made a convolution with the frequency response function of the sensor.

This analysis shows that an echo-impulse in the SR band is absent, while it is evident in the SRS band. The polarity of the secondary pulse is negative, i.e. opposite to the polarity of the main pulse. Because the SR is a sensitive indicator of the lightning discharge, the lack of its response during the occurrence of secondary pulse in the IAR band indicates that the latter one is not caused by secondary lightning discharges.

\section{Modeling Signals and Spectra}

Here we demonstrate that SRS indeed can be formed by a peculiar two-pulse structure of the disturbances produced by a local lightning activity (Fig. 8). The Fourier and wavelet power spectra of the paired modeling impulses, delayed by $\Delta t=1 \mathrm{~s}$ of similar (left-hand panel) and opposite (right-hand panel) polarities have been estimated. For the pair of impulses with the same polarity, the spectrum has spectral maxima at frequencies which are multiples of the 

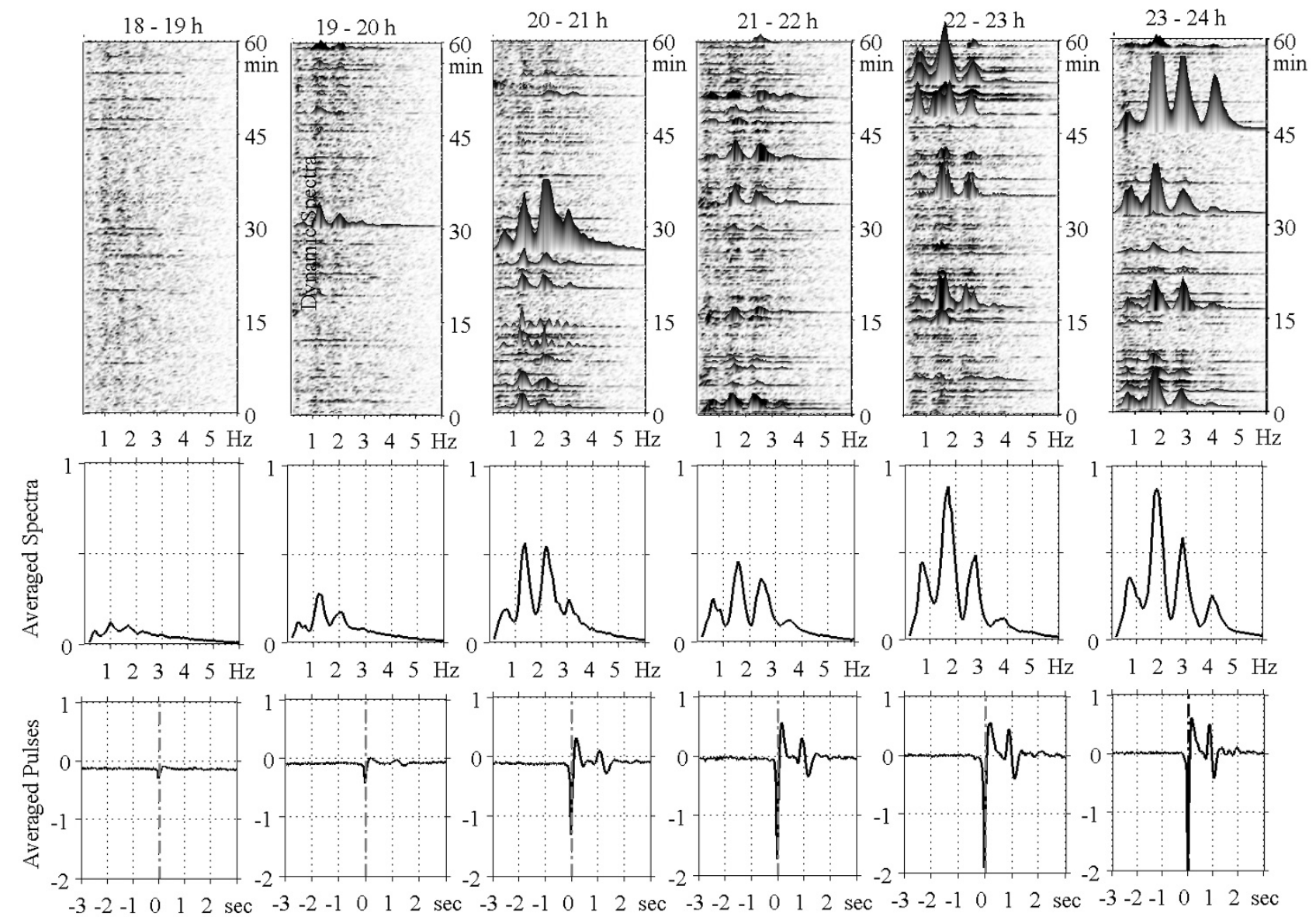

Fig. 6. The correspondence between the temporal evolution of the impulsive background and SRS for the 10/04 during the time interval $18-24$ LT ( $D$ component). The upper panels show hourly quasi-3D spectra in the range $0.1-6 \mathrm{~Hz}$. The middle panels show the averaged spectra for the hourly intervals corresponding to the upper panel. The bottom panels show the superposed waveforms of all recorded impulses.

inverse delay time between impulses, namely $1,2,3, \ldots \mathrm{Hz}$; whereas for impulses with opposite polarities at these frequencies the spectral minima are formed.

The SRS of the horizontal magnetic components in the range from 0.1 to $10 \mathrm{~Hz}$ is regularly observed at the ground for night-time conditions. Sometimes, up to 15 resonance spectral lines, extending even above the second SR frequency, were occasionally seen (Bösinger et al., 2002). In our opinion, this multi-spectral feature with a high number of harmonics may evidence the occurrence of strongly nonsinusoidal waveforms of impulses.

\section{Theoretical Scheme}

We suppose that the multi-frequency SRS can be formed due to the sequence of paired impulses. The main impulse is produced by a lightning discharge, whereas the second impulse is caused by the re ection of the main impulse from the IAR upper boundary. A possible scheme of the IAR excitation by a lightning is as follows (Fig. 9). An electric discharge generates an initial TH (or electric) mode pulse which propagates in the waveguide ionosphere-ground. The magnetic disturbance in this pulse is orthogonal to the direction towards a source. The initial pulse is also re ected from the bottom ionosphere. Upon this re ection TE mode is ex- cited owing to the mode coupling in the anisotropic lower ionosphere. Therefore, the re ected from the ionosphere pulse comprises additionally the magnetic component directed towards a source. Both direct and re ected pulses reach an observation site with a negligible delay for the IAR and SR frequency ranges. Meanwhile, the TH pulse partially penetrates into the ionosphere, travels up the ionosphere as an Alfven pulse, and re ects back from the upper IAR boundary. This pulse can return back to the ground as an echo-pulse in the magnetic (TE) mode owing to the mode conversion in the anisotropic lower ionosphere. A TH echo pulse cannot be detected on the Earth surface because of a low penetration of the electric mode into the highly resistive atmosphere. The delay between the echo pulse and the stroke (or main pulse) is about the time of the Alfven wave propagation up and down in the ionosphere. Thus, the pulse repetition period is about the IAR fundamental period.

However, this qualitative scheme is to be validated by a numerical modeling. In particular, this modeling should clarify whether the main contribution to the observed time pattern is produced by the region near the stroke, above the receiving station, or by an integral effect along the propagation path? 

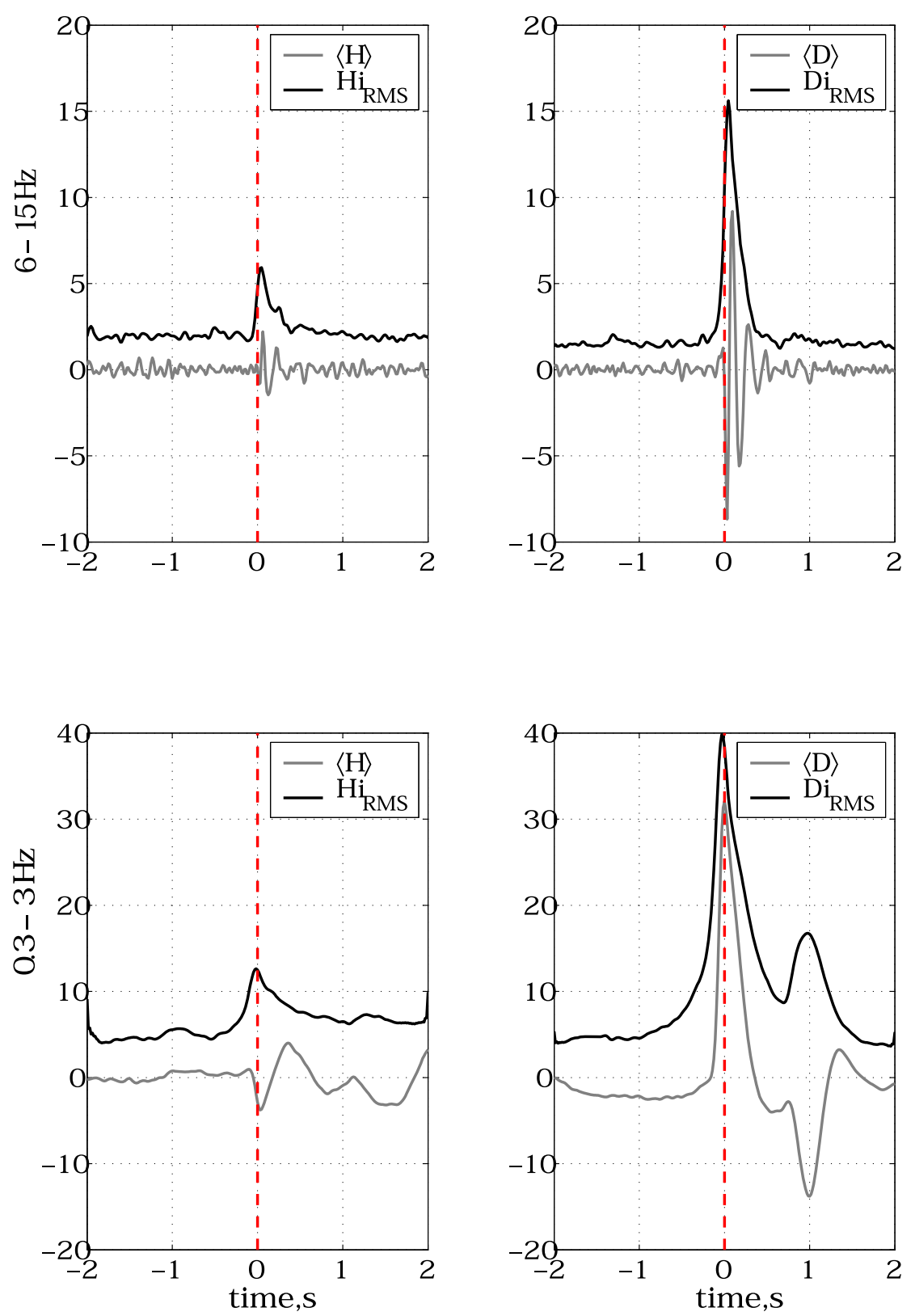

Fig. 7. Averaged field variations (grey line) and RMS of the instantaneous amplitude (dark line) for $H$ (left-hand panels) and $D$ (right-hand panels) components in arbitrary units. The reference moment for the superposed epoch analysis is shown by a vertical dotted line. Upper panels: magnetic response in SR 6-15 Hz (upper panel) and SRS 0.3-3 Hz (lower panel) bands on Oct. 04.

\section{Discussion}

It is generally believed that the global lightning activity concentrated in the world thunderstorm centers is the basic mechanism for excitation of the SR. However, an attempt to apply the same mechanism for the interpretation of SRS comes across with certain difficulties at middle latitudes far from the tropics. The ULF spectrum, calculated under the assumption of the distant world thunderstorms as a source, and experimental spectra agree in the SR band, but they disagree by $1-2$ orders of magnitude in the IAR frequency range (Fedorov et al., 2006). This fact suggests that the SRS is not simply due to a stochastic lightning discharge process of tropical thunderstorms, but that some generation mechanisms for the IAR excitation at middle latitudes should exist.

In our opinion, the SRS can be excited by regional thun- derstorms, within thousand $\mathrm{km}$. Along with the main pulse, the pulses reflected from the upper IAR boundary can be registered on the ground. The time delay between pulses is equal to the IAR resonant period. In many cases, two or even three pulses have been detected, but the main first pulse has been registered both in the SR and IAR bands, whereas the secondary pulses have been recorded in the IAR range only. At the same time we have to mention that though the statistical analysis undoubtedly reveals the occurrence of the secondary pulse, some individual magnetic pulses, alike the lightning-associated pulses, were not followed by any secondary pulse. The lack of a secondary pulse is hardly can be attributed to a sudden change of the IAR resonant properties, and needs an additional examination.

The polarization of the horizontal magnetic components 

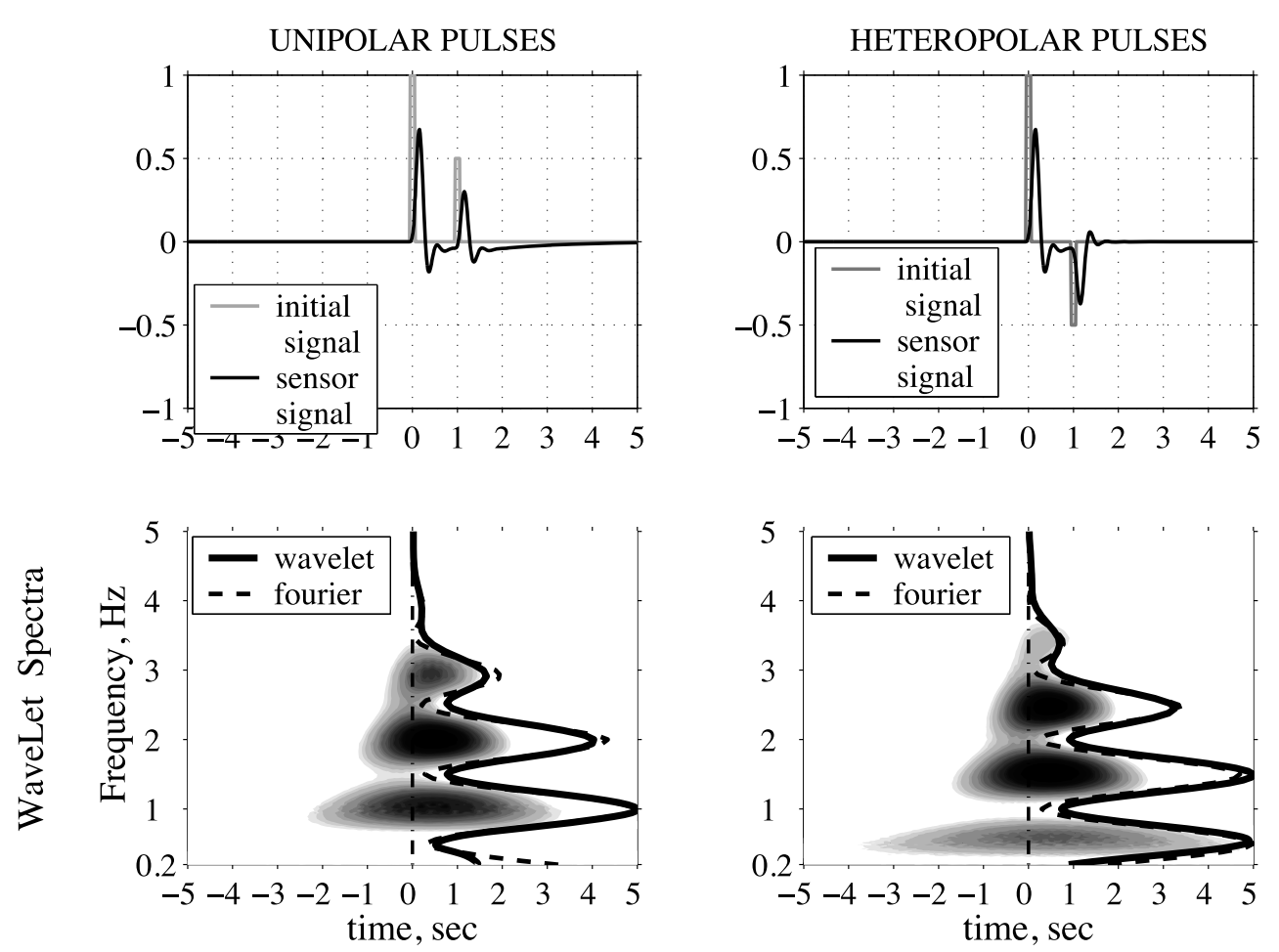

Fig. 8. The wavelet scalogram and Fourier power spectra of the artificial paired modeling impulses with the same polarity (left-hand bottom panels) and opposite polarity (right-hand bottom panel). The initial signal (upper panels) is mimicked as shown by the grey line, the acquisition system response with the account of the conversion function (Fig. 1) is denoted by dark line.

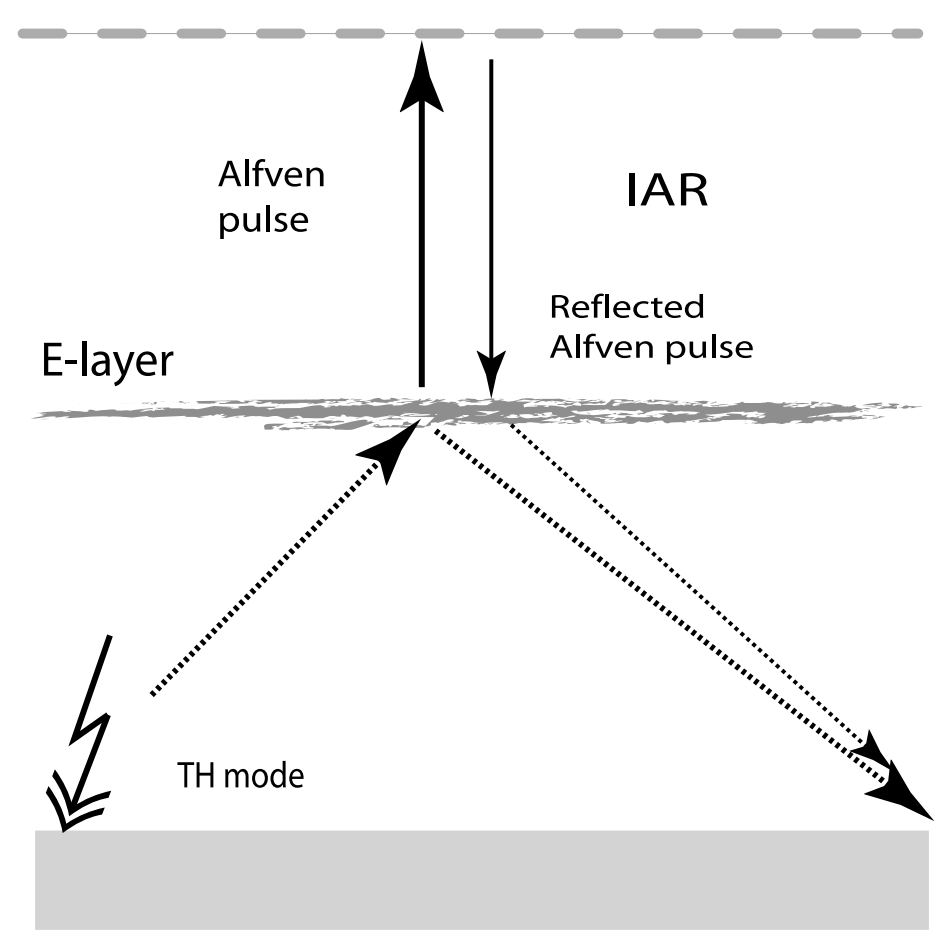

Fig. 9. A scheme of the IAR excitation by a lightning discharge and formation of the secondary echo-pulse observed on the ground.

of the main pulse conforms the assumption that this disturbance is produced by a vertical current associated to lightning. According to the simple theoretical expectations, the secondary pulse is to be polarized along the direction rotated by $\pi / 2$ from the main pulse polarization ellipse axis, because the secondary pulse is to be related to another wave mode. However, observations have shown that the differ- ence in the orientation of the polarization ellipses of the main and secondary pulses is rather small, not more that $30^{\circ}$. This fact needs a more detailed theoretical modeling.

In some events, rather surprisingly, no ULF magnetic response to intense regional strokes was detected. The lack of ULF response is possibly related to a low intensity of the continuing current in those discharges. Because we have no 
information about the intensity of the continuing current in our events, we could not validate this assumption, and this paradox needs a further examination.

The mechanism of the SRS origin owing to the regional thunderstorms cannot be considered as unique and the only one. The distant thunderstorm activity, probably, contributes to the ULF impulsive background, especially at low latitudes. The suggested hypothesis does not explain all the observational results, in particular, the seasonal variation of the SRS occurrence rate. The SRS observations at polar latitudes pose a problem, too (Semenova et al., 2008). So, the rate of the relative contribution from various possible mechanisms to the IAR stimulation is still an open experimental problem. Unfortunately, the information about the lightning occurrence and their location is available from VLF monitoring systems only on a commercial basis. Therefore, any scrutiny statistical study would be possible only when those data would be freely available to the scienti c community.

The question of the magnitudes of the thunderstorm related ULF elds in the upper ionosphere is rather controversial. Grei nger and Grei nger (1976) predicted only a weak strength of the ULF elds in the upper ionosphere above the thunderstorm. On the other hand, satellite observations sometimes detected very large ULF variations up to 1$10 \mathrm{mV} / \mathrm{m}$ (Burke et al., 1992). Discoveries of the transient luminous effects (TLE), such as elves, red sprites, and blue jets, possibly caused by the breakdown at altitudes $\sim 90 \mathrm{~km}$, revealed the possibility of a much stronger lightning coupling to the ionosphere than previously deemed possible. These processes, associated with large high-altitude nonlinear currents, may strongly enhance the weight of the ULF-ELF spectrum in the lightning transients in the ionosphere, and the IAR excitation by strong lightning discharge (Sukhorukov and Stubbe, 1997). However, so far few attempts (e.g., Bösinger et al., 2006) to reveal any speci c ULF response to distant high-altitude TLE as compared with ordinary lightning were not successful.

\section{Conclusion}

The statistical superposed epoch analysis has shown that magnetic disturbances during the regional lightning activity comprises regular series of paired pulses. An initial pulse caused by an atmospheric discharge is followed by 1-2 pulses with a regular time delay. This echo-pulse is not caused by some secondary stroke, because no simultaneous burst can be seen in the SR band. Such paired pulses results in the formation of the multi-band SRS in dynamic spectra. The observed time delay ts well the SRS structure. The secondary pulses are supposed to be produced by the re ection of the initial lightning pulse from the IAR upper boundary. Thus, regional thunderstorms can give a noticeable input to the IAR power spectrum, and may be responsible, at least partly, for the SRS occurrence at middle latitudes.

Acknowledgments. We thank H. Hamaguchi, Y. Katoh, M. Satoh, Y. Yamamoto, Y. Ikegami, and M. Sera of the SolarTerrestrial Environment Laboratory, Nagoya University, for their helpful support in the development and operation of the induction magnetometer at Moshiri. This work was supported by Grantsin-Aid for Scienti c Research (18403011 and 20244080) by the
MEXT of Japan, and grant 10-05-93107 from RFBR. Constructive criticism of reviewers is appreciated.

\section{References}

Belyaev, P. P., S. V. Polyakov, V. O. Rapoport, and V. Y. Trakhtengertz, Discovery of the resonance spectrum structure of atmospheric electromagnetic noise background in the range of short-period geomagnetic pulsations, Dokl. Akad. Nauk SSSR, 297, 840, 1987.

Belyaev, P. P., S. V. Polyakov, V. O. Rapoport, and V. Y. Trakhtengertz, The ionospheric Alfven resonator, J. Atmos. Terr. Phys., 52, 781, 1990.

Belyaev, P. P., T. Bösinger, S. V. Isaev, and J. Kangas, First evidence at high latitudes for the ionospheric Alfven resonator, J. Geophys. Res., 104, 4305, 1999.

Bösinger, T., C. Haldoupis, P. P. Belyaev, M. N. Yakunin, N. V. Semenova, A. G. Demekhov, and V. Angelopoulos, Spectral properties of the ionospheric Alfven resonator observed at a low-latitude station $(\mathrm{L}=1.3), J$. Geophys. Res., 107, 1281, doi:10.1029/2001JA005076, 2002.

Bösinger, T., A. Mika, S. L. Shalimov, C. Haldoupis, and T. Neubert, Is there a unique signature in the ULF response to sprite-associated lightning ashes?, J. Geophys. Res., 111, NA10, 2006.

Burke, W. J., T. L. Aggson, N. C. Maynard, W. R. Hoegy, R. A. Hoffman, R. M. Candy, C. Liebrecht, and E. Rodgers, Effects of a lightning discharge detected by the DE 2 satellite over hurricane Debbie, J. Geophys. Res., 97, 6359-6367, 1992.

Demekhov, A. G., V. Y. Trakhtengertz, and T. Bösinger, Pc 1 waves and ionospheric Alfven resonator: generation or ltration?, Geophys. Res. Lett., 27, 3805-3808, 2000.

Fedorov, E. N., A. J. Schekotov, O. A. Molchanov, M. Hayakawa, V. V. Surkov, and V. A. Gladishev, An energy source for the mid-middle latitudes IAR: World thunderstorm centers, nearby discharges or neutral wind uctuations?, Phys. Chem. Earth, 31, 462-468, 2006.

Fowler, R. A., B. J. Kotick, and R. D. Elliot, Polarization analysis of natural and arti cially induced geomagnetic micropulsations, J. Geophys. Res., 72, 2871-2883, 1967.

Fraser-Smith, A. C., ULF magnetic elds generated by electrical storms and their signi cance to geomagnetic pulsation generation, Geophys. Res. Lett., 20, 467-470, 1993.

Fujita, S. and T. Tamao, Duct propagation of hydromagnetic waves in the upper ionosphere. 1. Electromagnetic eld disturbances in high latitudes associated with localized incidence of a shear Alfven wave, J. Geophys. Res., 93, 14665-14673, 1988.

Grei nger, C. and P. Grei nger, Transient ULF electric and magnetic elds following a lightning discharge, J. Geophys. Res., 81, 2237-2247, 1976.

Lysak, R. L., Feedback instability of the ionospheric resonator cavity, $J$. Geophys. Res., 96, 1553, 1991.

Molchanov, O. A., A. Yu. Schekotov, E. N. Fedorov, and M. Hayakawa, Ionospheric Alfven resonance at middle latitudes: Results of observations at Kamchatka, Phys. Chem. Earth, Parts A/B/C, 29, 649-655, 2004.

Nickolaenko, A. P. and M. Hayakawa, Resonances in the Earth-Ionosphere cavity, Klumer Academic Publishers, Dordrecht/Boston/London, 2002.

Pilipenko, V., E. Fedorov, K. Mursula, and T. Pikkarainen, Generation of magnetic noise bursts during distant rocket launches, Geophysica, 41(12), 57-72, 2005.

Pokhotelov, O. A., V. Khruschev, M. Parrot, S. Senchenkov, and V. P. Pavlenko, Ionospheric Alfven resonator revisited: Feedback instability, J. Geophys. Res., 106, 813, 2001.

Polyakov, S. V. and V. O. Rapoport, The ionospheric Alfven resonator, Geomagn. Aeron., 21, 610-614, 1981.

Prikner, K., K. Mursula, F. Z. Feygin, J. Kangas, R. Kerttula, T. Pikkarainen, O. A. Pokhotelov, and V. Vagner, Non-stationary Alfvén resonator: vertical pro les of wave characteristics, J. Atmos. Sol.-Terr. Phys., 62, 311-322, 2000.

Semenova, N. V., A. G. Yahnin, A. N. Vasil'ev, and O. Amm, Speci c features of resonance structures in spectra of ULF electromagnetic noise at high latitudes (Barentsburg observatory), Geomagn. Aeron., 48, 3644, 2008.

Shalimov, S. and T. Bösinger, On distant excitation of the ionospheric Alfven resonator by positive cloud-to-ground lightning discharges, $J$. Geophys. Res., 113, 2008.

Shiokawa, K., R. Nomura, K. Sakaguchi, Y. Otsuka, Y. Hamaguchi, M. Satoh, Y. Katoh, Y. Yamamoto, B. M. Shevtsov, S. Smirnov, I. Poddelsky, and M. Connors, The STEL induction magnetometer network for observation of high-frequency geomagnetic pulsations, Earth Planets Space, 62, 517-524, 2010. 
Sukhorukov, A. I. and P. Stubbe, Excitation of the ionospheric Alfven resonator by strong lightning discharges, Geophys. Res. Lett., 24, 829832, 1997.

Surkov, V. V., O. A. Pokhotelov, M. Parrot, E. N. Fedorov, and M. Hayakawa, Excitation of the ionospheric resonance cavity by neutral winds at middle latitudes, Ann. Geophys., 22, 2877-2889, 2004.

Surkov, V. V., M. Hayakawa, A. Y. Schekotov, E. N. Fedorov, and O. A. Molchanov, Ionospheric Alfven resonator excitation due to nearby thunderstorms, J. Geophys. Res., 111, A01303, 2006.

Trakhtengertz, V. Yu. and A. Ya. Feldstein, Turbulent Alfven boundary layer in the polar ionosphere, 1, Excitation conditions and energetics, $J$. Geophys. Res., 96, 363, 1991.

Trakhtengerts, V. Y., A. C. Demekhov, P. P. Belyaev, S. V. Polyakov, E.
N. Ermakova, and S. V. Isaev, A mechanism of anticorrelation in the occurrence of ULF electromagnetic noise resonance structure and Pc1 magnetic pulsations through the solar activity cycle, J. Atmos. Sol.-Terr. Phys., 62, 253-256, 2000.

Yahnin, A. G., N. V. Semenova, A. A. Ostapenko, J. Kangas, J. Manninen, and T. Turunen, Morphology of the spectral resonance structure of the electromagnetic background noise in the range of $0.1-4 \mathrm{~Hz}$ at $\mathrm{L}=5.2$, Ann. Geophys., 21, 779, 2003.

A. Schekotov, V. Pilipenko (e-mail: pilipenko_va@mail.ru), K. Shiokawa, and E. Fedorov 